\title{
DEPRESSIVE AND ANXIETY DISORDERS OF BREAST CANCER PATIENTS IN SURVIVORSHIP \\ Po-Hsien Lin ${ }^{1}$, Yong Alison Wang ${ }^{2}$
}

1. Department of Psychiatry, Koo Foundation Sun Yat-Sen Cancer Center (KF-SYSCC), Taipei, Taiwan 2. Department of Internal Medicine, Koo Foundation Sun Yat-Sen Cancer Center (KF-SYSCC), Taipei, Taiwan

\section{Objectives:}

To explore the characteristics of mood disorders and the associations with physical symptoms in breast cancer survivors.

\section{Background and aims:}

Breast cancer patients in survivorship still suffered from many physical symptoms which derive from previous anti-cancer treatments, current anti-hormone therapy and aging/menopause related change. We aimed to investigate their mood disorders and their relationship to physical symptoms.

Materials and methods:

Breast cancer survivors were evaluated approximately 1 year after cancer surgery. Demographic data including age, education level, marital status, employment, history of substance use(cigarettes and alcohol), staging of cancer and treatments were collected by research assistants. Mood disorders were assessed using Patient Health Questionnaire (PHQ-9) and Generalized Anxiety Disorder (GAD-7) Questionnaires. We used a cut-off score of 10 or more on the PHQ-2 and GAD-2 as indicators of significant depression and anxiety disorders. Physical symptoms for breast cancer survivors were collected using the Breast Cancer Prevention Trial (BCPT) Symptom Scale. We used cut-off score of 2 or more as indicators of significant somatic symptoms. Chi-square test or Fisher's exact test (with an expected frequency of five or less) and logistic regression were performed to explore the risk factors of depressive and anxiety disorder, and analyses were performed by SAS 9.4 .

Results:

Among 542 breast cancer survivors included in the analysis, $34(6.27 \%)$ had depressive disorder and 40 (7.38\%) had anxiety disorder. History of cigarettes use, vasomotor symptoms, vaginal dryness/dyspareunia, musculoskeletal pain, cognitive impairment, weight problem and arm edema/limitation of movement were associated with depressive disorder in the univariate analysis. On the other hand, vasomotor symptoms, musculoskeletal pain, cognitive impairment and arm edema/limitation of movement were associated with anxiety disorder in the univariate analysis. Using multivariate logistic regression analysis, smoking (AOR=3.33, 95\% C.I.= 1.12-9.88), musculoskeletal pain (AOR=6.09, 95\% C.I.=2.44-15.20), cognitive impairment (AOR=11.80, 95\% C.I.=4.96-28.09), and weight problems (AOR=4.87, 95\% C.I.=1.89-12.55) predicted the presence of depressive disorder; vasomotor symptoms(AOR=2.44, 95\% C.I.= 1.04-5.72), musculoskeletal pain (AOR=5.03, 95\% C.I.=2.26-11.22), and cognitive impairment (AOR=7.24, 95\% C.I.=3.21-16.32) predicted the presence of anxiety disorder. Conclusions:

Mental disorder and physical symptoms are highly related and sometimes may have the same causes in cancer survivors. Comprehensive assessment and patient care for cancer survivors are necessary to improve their quality of life.

E-mail Address:

phlin@kfsyscc.org
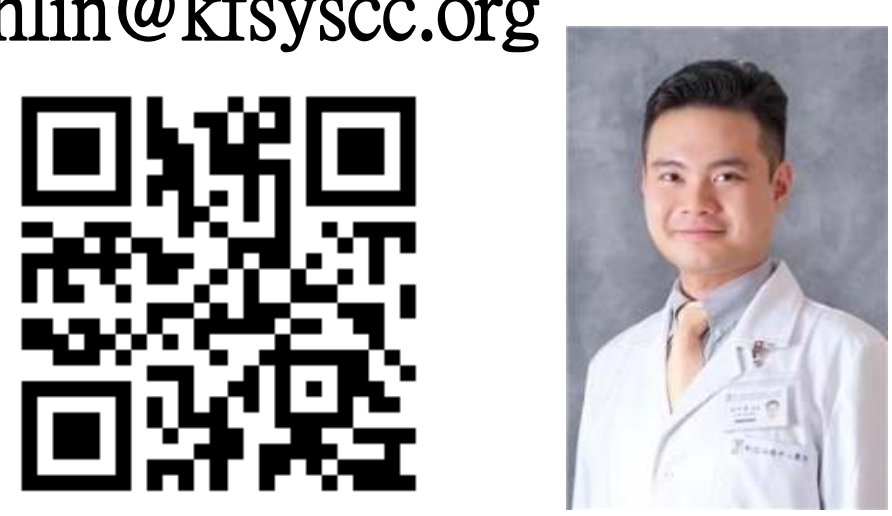

Table. 2 Model-based adjusted odds ratio (aOR) by logistic regression analysis with the backward method

\begin{tabular}{|r|r|r|r|}
\hline Variable & $\begin{array}{r}\text { Beta } \\
\text { Coefficient }\end{array}$ & $\mathrm{aOR}$ & $\begin{array}{r}95 \% \\
\text { Confidence Interval }\end{array}$ \\
\hline Depressive Disorder & & & \\
\hline Smoking & 0.6013 & 3.329 & $1.121-9.882$ \\
\hline Musculoskeletal pain & 0.9036 & 6.093 & $2.442-15.202$ \\
\hline Cognitive impairment & 1.2342 & 11.803 & $4.960-28.089$ \\
\hline Weight Problem & 0.7918 & 4.872 & $1.891-12.554$ \\
\hline Anxiety Disorder & & & \\
\hline Vasomotor symptoms & 0.4455 & 2.437 & $1.039-5.717$ \\
\hline Musculoskeletal pain & 0.8081 & 5.034 & $2.259-11.215$ \\
\hline Cognitive impairment & 0.9900 & 7.242 & $3.213-16.324$ \\
\hline
\end{tabular}

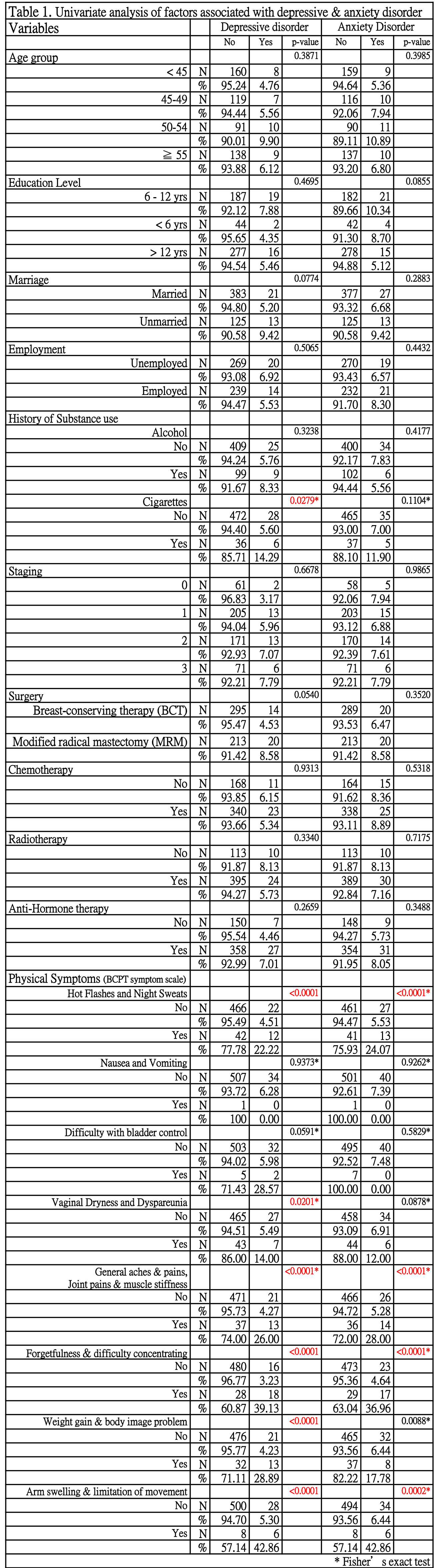

